\title{
Recommendation for Cardiac Magnetic Resonance Imaging-Based Phenotypic Study: Imaging Part
}

\author{
Chengyan Wang ${ }^{1}\left(\mathbb{D} \cdot\right.$ Yan $\mathrm{Li}^{2} \cdot$ Jun $\mathrm{Lv}^{3} \cdot{\text { Jianhua } \mathrm{Jin}^{4} \cdot \text { Xumei Hu}}^{1} \cdot$ Xutong Kuang $^{1} \cdot$ Weibo Chen $^{5} \cdot \mathrm{He} \mathrm{Wang}^{1,6,7}$
}

Received: 13 February 2021 / Revised: 22 May 2021 / Accepted: 25 May 2021 / Published online: 28 July 2021

(C) International Human Phenome Institutes (Shanghai) 2021

\begin{abstract}
Cardiac magnetic resonance (CMR) imaging provides important biomarkers for the early diagnosis of many cardiovascular diseases and has been reported to reveal phenome-wide associations of cardiac/aortic structure and functionality in population studies. Nevertheless, due to the complexity of operation and variations among manufactural vendors, magnetic field strengths, coils, sequences, scan parameters, and image analysis approaches, CMR is rarely used in large cohort studies. Existing guidelines mainly focused on the diagnosis of cardiovascular diseases, which did not aim to basic research. The purpose of this study was to propose a recommendation for CMR based phenotype measurements for cohort study. We classify the imaging sequences of CMR into three categories according to the importance and universality of corresponding measurable phenotypes. The acquisition time and repeatability of the phenotypic measurement were also taken into consideration during the categorization. Unlike other guidelines, this recommendation focused on quantitative measurement of large amount of phenotypes from CMR.
\end{abstract}

Keywords Cardiac imaging $\cdot$ Magnetic resonance imaging $\cdot$ Phenotype $\cdot$ Standardization

Chengyan Wang

wangcy@fudan.edu.cn

$\triangle$ He Wang

hewang@fudan.edu.cn

1 Human Phenome Institute, Fudan University, 825

Zhangheng Road, Pudong New District, Shanghai 201203,

China

2 Department of Radiology, Ruijin Hospital, Shanghai Jiao Tong University School of Medicine, Shanghai, China

3 School of Computer and Control Engineering, Yantai University, Yantai, China

4 School of Data Science, Fudan University, Shanghai, China

5 Philips Healthcare. Co., Shanghai, China

6 Institute of Science and Technology for Brain-Inspired Intelligence, Fudan University, 220 Handan Road, Yangpu District, Shanghai 200433, China

7 Key Laboratory of Computational Neuroscience and Brain-Inspired Intelligence (Fudan University), Ministry of Education, Shanghai, China

\section{Introduction}

Cardiac imaging can provide a variety of biomarkers for the early diagnosis of cardiovascular diseases and has been reported to uncover phenome-wide associations of cardiac/ aortic structure and functionality in population studies (Nakamura and Sadoshima 2018; Jaffe et al. 2006; Bai et al. 2020). Due to the development in technology, cardiac imaging has revealed an increasing number of phenotypes. Generally speaking, cardiac imaging techniques include angiocardiography, ultrasound (US), isotope imaging, computed tomography (CT), and cardiac magnetic resonance (CMR) imaging. Among them, CMR provides a non-invasive cardiovascular functionality assessment, with high soft-tissue contrast, high repeatability, and without producing radiation (Hundley et al. 2010; Finn et al. 2006). The European Society of Cardiology (ESC) has recommended CMR as the gold standard for assessing ventricular volumes and myocardial wall motions (Wassmuth and Schulz-Menger 2011). Although CMR has an important role in clinical examinations, it is still not widely used in large cohort studies due to the complexity of operation and variations among manufactural vendors, magnetic field strengths, coils, sequences, scan parameters, and image analysis approaches. Therefore, 
there is an urgent need to establish a standardized CMR protocol, as well as a data analysis framework to ensure robust phenotypic analysis.

Currently, there are several recommendations and consensus on clinical CMR studies. The Society for CMR (SCMR) has launched a standardized CMR program in 2008 (Kramer et al. 2008), which was updated in 2013 and 2020 (Kramer et al. 2013, 2020). The International Consensus Group (ICG) on CMR Diagnosis of Myocarditis proposed a standardized imaging and reporting criteria for myocarditis patients in 2009 (Friedrich et al. 2009). In 2010, the American Heart Association (AHA), American College of Cardiology (ACC), American College of Radiology (ACR), and SCMR Expert Panel provided consensus recommendations to standardize the technological and operational requirements of CMR imaging and its application in cardiovascular diseases (Hundley et al. 2010). The Journal of the American College of Cardiology (JACC) Scientific Expert Panel provided a consensus on CMR diagnostic criteria for myocardial inflammation in patients with suspected acute or active myocardial inflammation in 2018 (Ferreira et al. 2018). In 2019, the Korean Society of Cardiovascular Imaging (KSCI) launched a CMR guideline including the equipment, sequence terminology, imaging planes, and drug usage for Asian populations (Jo et al. 2019). More recently, the SCMR announced specific recommendations on using CMR protocols in the era of the COVID-19 pandemic (Kelle et al. 2020).

The abovementioned guidelines/consensus are mainly focused on the clinical diagnosis of cardiovascular diseases, and did not aim to basic research in the field of life sciences. Generally speaking, a large cohort study has the following characteristics: (a) quantitative measurements are required instead of large amounts of images; (b) the phenotypes obtained from CMR should be reliable and repeatable among different equipments; (c) non-contrast-enhanced imaging is preferred since the targeting population is often made up of volunteers; (d) the requirements and time budgets are flexible for different studies, which makes it necessary to provide diversified imaging options for selection. Herein, we proposed a recommendation for CMR-based phenotype measurements according to the special considerations for cohort studies. Our recommendation also referred to the clinical guidelines/consensus mentioned above as well as the scan protocols used in the UK Biobank Project (Henningsson et al. 2020). We classified the currently available CMR imaging sequences into three categories according to the importance and universality of corresponding phenotypes. The contrast-enhanced imaging sequences were treated as Category III plan due to their relatively low acceptance by participants. Careful considerations of the acquisition efficiency, stability, and repeatability of the phenotype were also made during the categorization. The CMR-derived phenotypes were divided into structural, functional, tissue characteristics, hemodynamics, and metabolic measurements. Unlike other guidelines, this recommendation aims to extract large amount of quantitative phenotypes from the commonly used CMR imaging protocols.

\section{Equipment and Preparation}

\section{Magnetic Field Strength}

Cardiac imaging can be performed on $1.5 \mathrm{~T}$ as well as $3.0 \mathrm{~T}$ scanners. Since a 3.0 T scanner is able to provide higher signal-to-noise ratio (SNR), and high magnetic field is recommended to be used as long as robust shimming and radiofrequency adjustment are available (Kramer et al. 2013).

\section{Coils}

Phased-array surface coils dedicated to cardiac imaging, with more than eight coil elements, are recommended. Abdominal phased-array coils can also be used as a second option.

\section{Preparation}

Adequate patient preparation before MRI scan is mandatory. Checklists include MR indications, contraindications, informed consent, fasting, food, and medications. Participants should be placed in a supine position on the table before scans. Respiratory detectors are placed on the lower chest. Electrodes should be attached as required. Electrocardiogram (ECG) signal should be checked before the scan.

\section{Imaging Planes}

The most commonly used imaging planes include twochamber $(2 \mathrm{CH})$, left ventricular (LV) outflow tract (LVOT or $3 \mathrm{CH}$ ), four-chamber (4CH), and short-axis (SA) (Fig. 1). The recommended order of scan planes is as follows:

1. Localizer imaging with multi-stack views.

2. SA imaging based on the vertical and horizontal long axis (LA) views. The position line should be perpendicular to the interventricular septum. The scan range is from the bottom to the apex of the heart, including the entire left ventricle.

3. 4CH imaging based on the vertical LA and the SA views. The position line should be orthogonal to the vertical LA images passing through the LV apex and center of the mitral valve.

4. $2 \mathrm{CH}$ imaging based on $4 \mathrm{CH}$ and $\mathrm{SA}$ views. The position line passes through the center of LV and parallel to the 

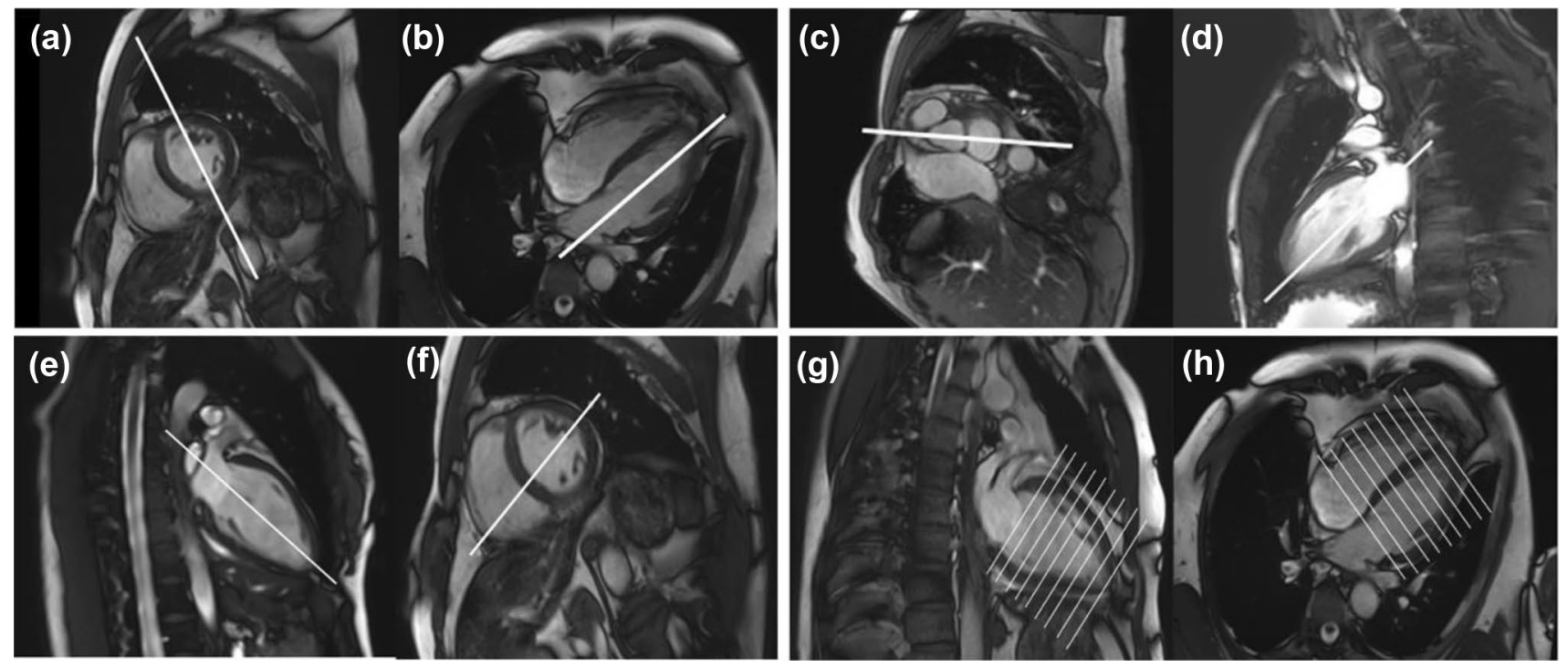

Fig. 1 Examples of the selections for cardiac imaging views, including $\mathbf{a}, \mathbf{b}$ two-chamber; $\mathbf{c}, \mathbf{d}$ left ventricular outflow tract; $\mathbf{e}, \mathbf{f}$ four-chamber and $\mathbf{g}, \mathbf{h}$ short-axis views

ventricular septum. The scan range is from the apex to the midpoint of the mitral valve.

5. 3CH imaging (LVOT) based on the shortest axis of the $\mathrm{LV}$ and parallel to the connection between the LV cavity and the aortic root.

\section{CMR Sequences}

CMR includes contrast-enhanced and non-contrast-enhanced protocols. Herein, we divided the CMR sequences into three categories according to their acquisition time, the importance of the corresponding phenotypes, measurement stability and repeatability. Most volunteers in cohort studies prefer noncontrast-enhanced protocols. Therefore, we categorized the contrast-enhanced sequences into Category III. For most studies, it is recommended to implement the Category I protocol $(<20 \mathrm{~min})$. If there is enough time, it is highly recommended to perform both Categories I and II protocols in a single study ( $<45 \mathrm{~min}$ ). The Category III protocol provided some measurements for studies with special requirements. The scan time will be more than one hour to perform the Category III sequences. It should be noted that all the sequences with the requirement of contrast agent injection were classified as Category III. The imaging sequences, contrast, parameters, and the corresponding phenotypes are illustrated in Tables 1 and 2.

\section{Category I}

\section{Black Blood Imaging}

a) Sequences: the most commonly used sequences for black blood imaging include the turbo spin-echo (TSE), the double inversion recovery (DIR) half-Fourier acquisition single-shot TSE (HASTE), and the triple inversion recovery (TIR) HASTE. The steady-state precession (TrueFISP) readout can also be considered if robust shimming can be achieved (Henningsson et al. 2020).

b) Scan parameters: $\mathrm{SA}$ and $4 \mathrm{CH}$ views are collected with the spatial resolution $\leq 2.0 \times 2.0 \mathrm{~mm}^{2}$, slice thickness is $\leq 8.0 \mathrm{~mm}$, and slice gap $\leq 4.0 \mathrm{~mm}$. Signals are collected at the end of the diastole with ECG trigger on.

c) Contrast: black blood imaging is a routine scan proto$\mathrm{col}$ in the morphological examination of the heart. The TSE sequence suppresses the blood flow signal by taking advantage of the flow effect of blood, while the DIRHASTE and TIR-HASTE sequences suppress the blood flow signal by applying inversion pulses to enhance the contrast between the myocardium and the background. Thus, the blood pool signal is low, while the myocardium signal is relatively high (Fig. 2a-d). Black blood 


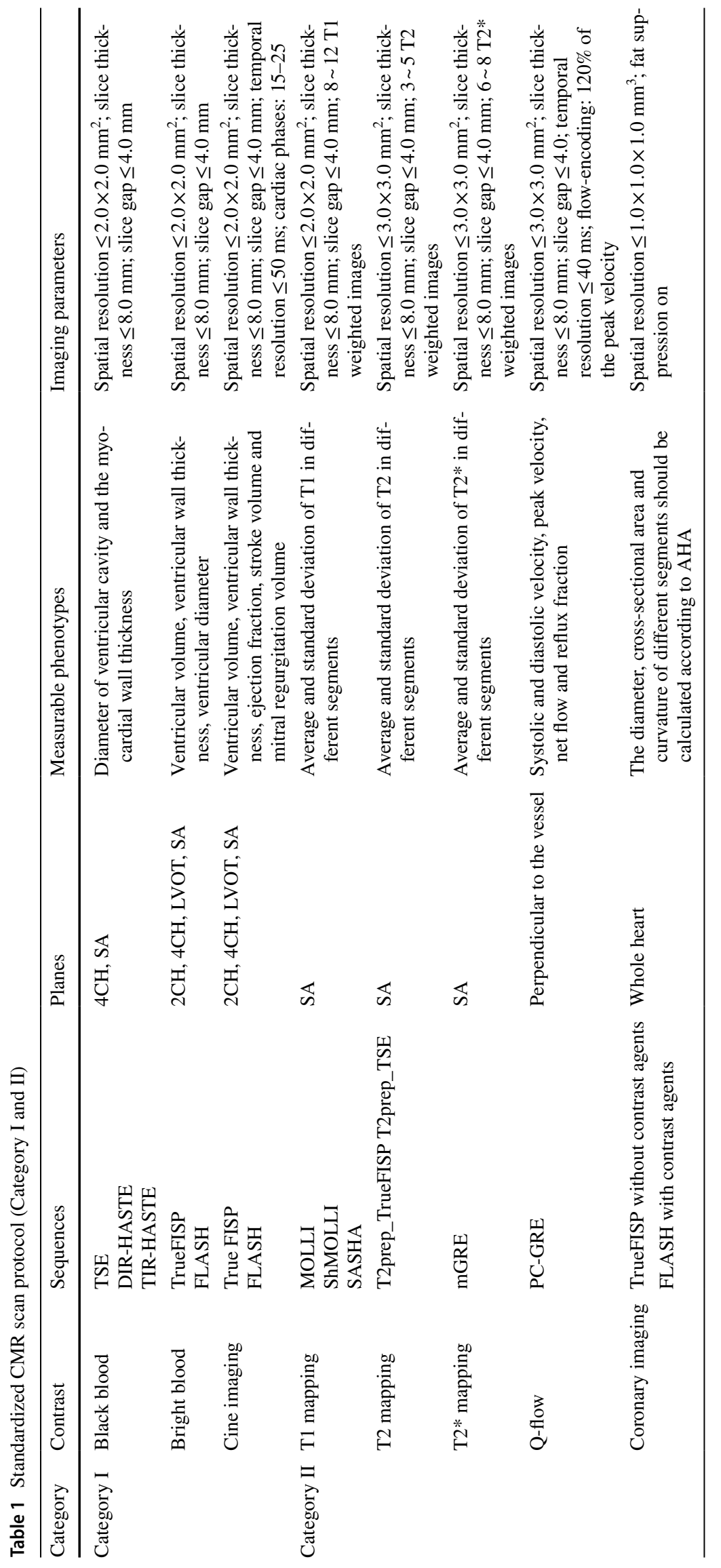




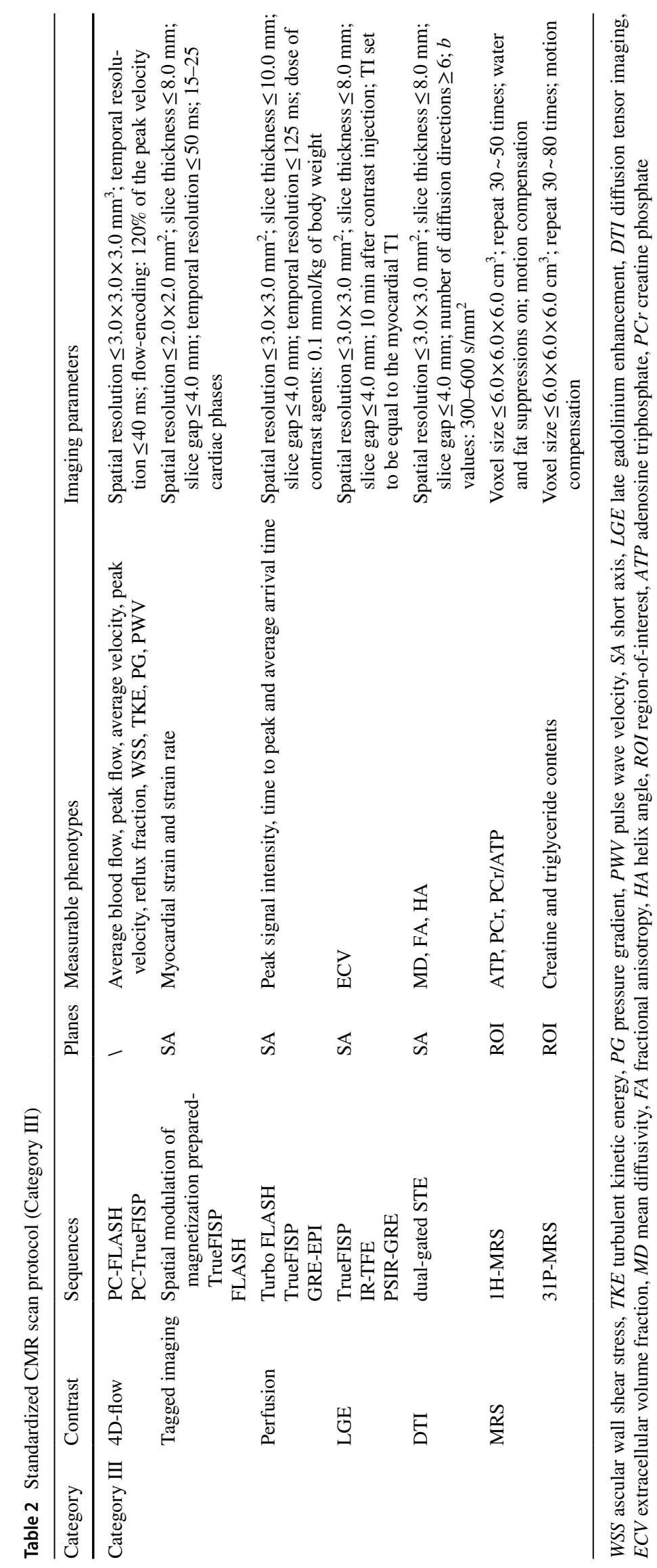



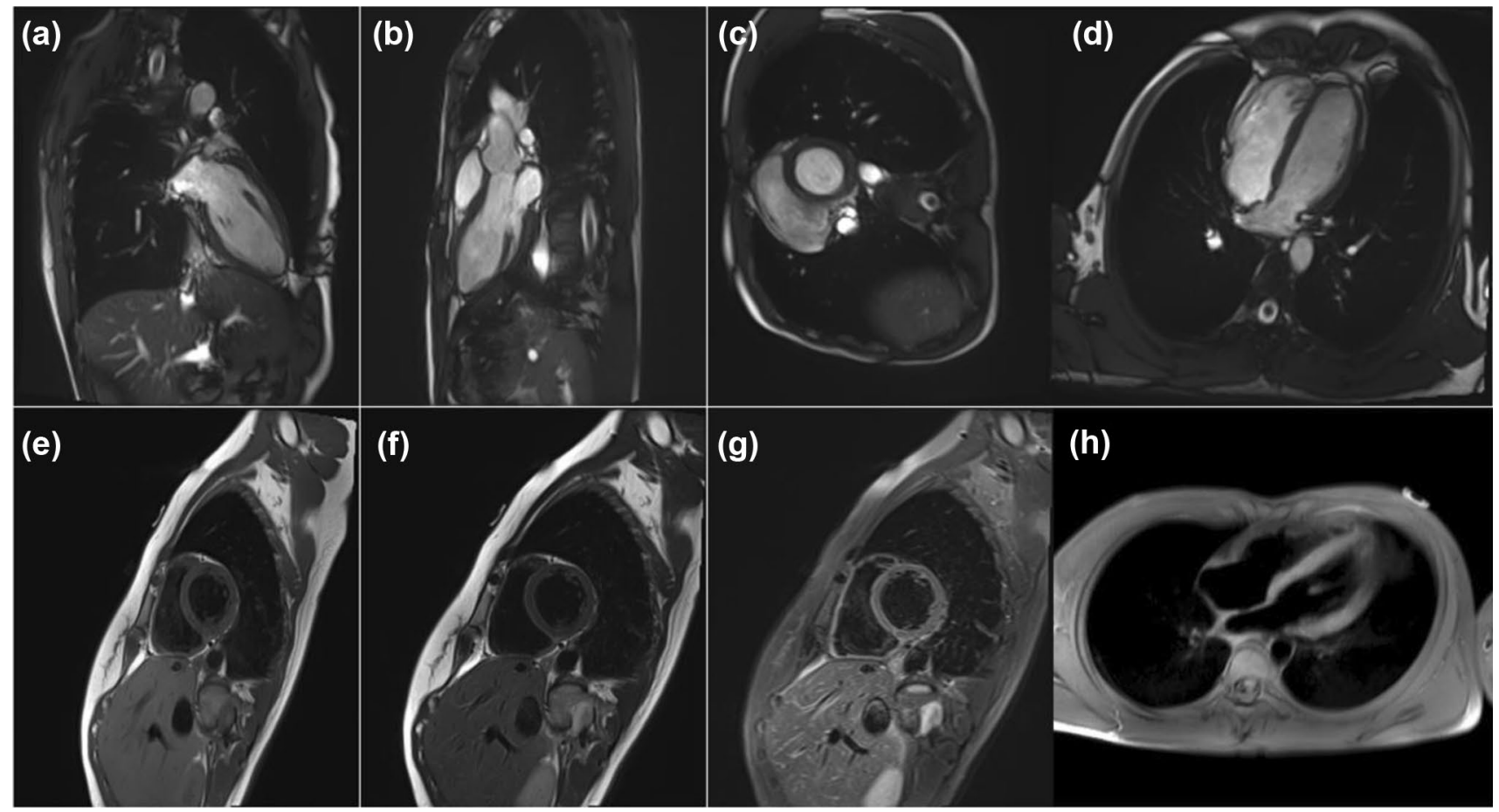

(h)

Fig. 2 Examples of a-d bright blood (different views) and black blood imaging with $\mathbf{e}$ T1-weighted, $\mathbf{f}$ T2-weighted, $\mathbf{g}$ fat suppression short-axis view, and $\mathbf{h}$ fat suppression $4 \mathrm{CH}$ view

imaging plays an important role in the detection of abnormal myocardial and pericardial signals.

d) Corresponding phenotypes: the phenotypes that can be extracted from black blood imaging include the ventricular volume, the diameter of the ventricular cavity, and the myocardial wall thickness.

\section{Bright Blood Imaging}

a) Sequences: the most commonly used sequences for bright blood imaging are the TrueFISP and the fast lowangle shot (FLASH) (Jo et al. 2019). It is recommended to apply TrueFISP on $1.5 \mathrm{~T}$ scanners to obtain higher SNR. TrueFISP is also recommended to be applied on $3.0 \mathrm{~T}$ scanners if the shimming is robust.

b) Scan parameters: $2 \mathrm{CH}, \mathrm{LVOT}, 4 \mathrm{CH}$, and SA views are collected with spatial resolution $\leq 2.0 \times 2.0 \mathrm{~mm}^{2}$, slice thickness $\leq 8.0 \mathrm{~mm}$, and slice gap $\leq 4.0 \mathrm{~mm}$. Signals are collected at the end of the diastole with ECG trigger on.

c) Contrast: bright blood imaging can provide high contrast between blood pool and myocardium without using a contrast agent (Fig. 2e, f). The bright blood imaging is often combined with cine imaging to achieve a one-stop assessment of the cardiac structure and functionality.

d) Corresponding phenotypes: similar to black blood imaging, the phenotypes of the ventricular volume, the diameter of the ventricular cavity, and the myocardial wall thickness can also be extracted from bright blood imaging.

\section{Cine Imaging}

a) Sequences: the most commonly used sequences for cine imaging are the segmented TrueFISP and the FLASH. For participants with regular heart rates, a multi-phase segmented scan is recommended for better image quality, while for patients with arrhythmia, real-time cine scan can be applied.

b) Scan parameters: $2 \mathrm{CH}$, LVOT, $4 \mathrm{CH}$, and SA views can be collected, with spatial resolution $\leq 2.0 \times 2.0 \mathrm{~mm}^{2}$, slice thickness $\leq 8.0 \mathrm{~mm}$, and slice gap $\leq 4.0 \mathrm{~mm}$. The cardiac cycle is usually segmented into $15-25$ phases with a temporal resolution $\leq 50 \mathrm{~ms}$.

c) Contrast: a series of cardiac images with different phases can be obtained. The bright blood dynamic images are usually collected as they can clearly observe the structural changes, and can be used to evaluate the systolic and diastolic functions of the heart. Typical cardiac cine images are shown in Fig. 3.

d) Corresponding phenotypes: the phenotypes extracted from cine imaging include ventricle and atrial volume, and myocardial wall thickness during the cardiac cycle. Some functionality characteristics including ejection 


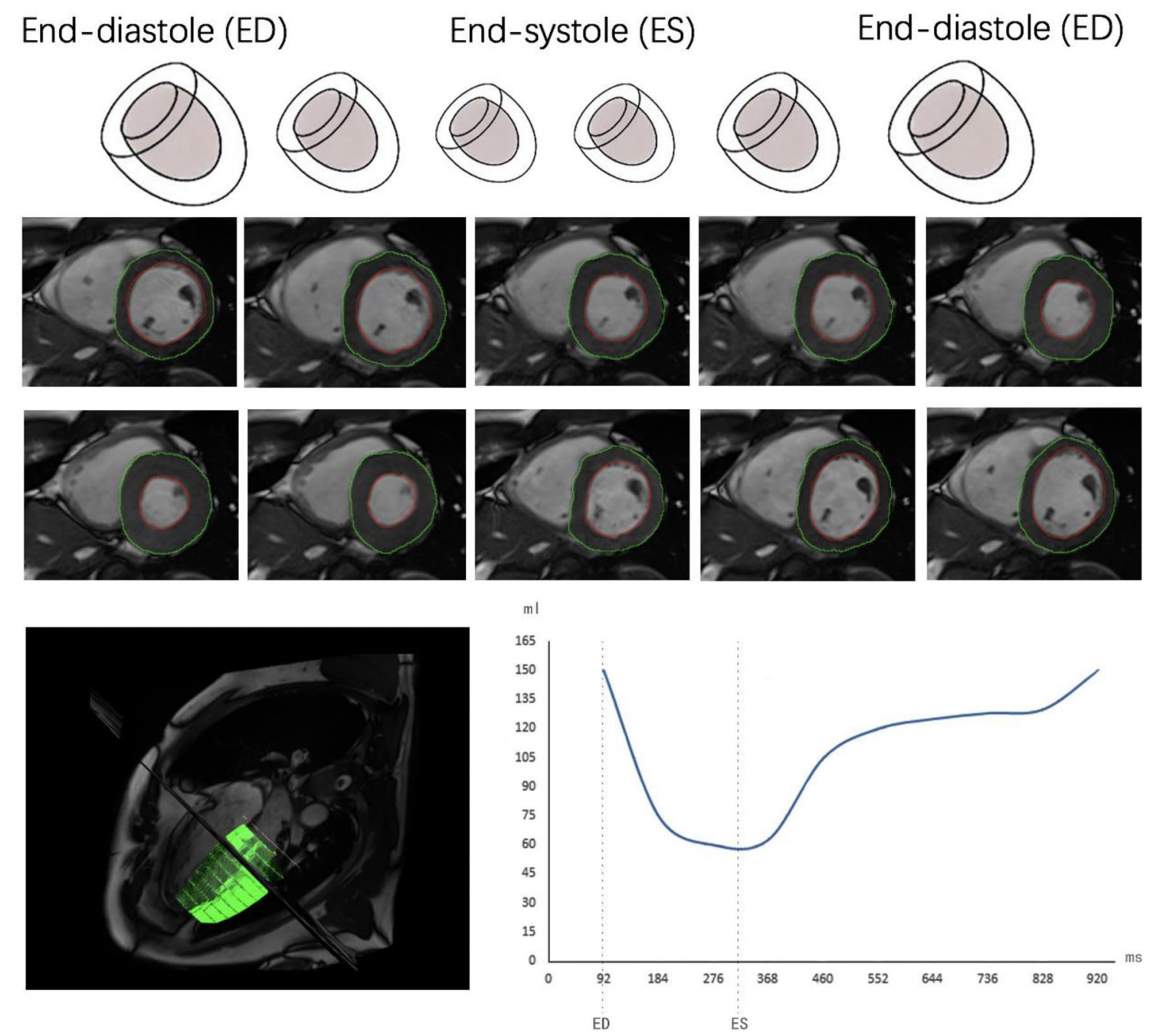

Fig. 3 Example of cardiac cine imaging during a cardiac cycle and the corresponding left ventricle volume measurements

fraction, cardiac output, stroke volume, and mitral regurgitation volume can also be obtained.

\section{Category II}

\section{T1 Mapping}

a) Sequences: the most commonly used sequences for $\mathrm{T} 1$ mapping include modified look-locker inversion recovery (MOLLI) (Messroghli et al. 2004), shorten MOLLI (shMOLLI) (Piechnik et al. 2010), and saturation recovery single-shot acquisition (SASHA) (Chow et al. 2014). Over recent years, several improvements have been made (Salerno et al. 2013; Weingärtner et al. 2014). Among the above-mentioned sequences, MOLLI is the most widely used one and is available on most clinical scanners. Compared with the other two techniques, the
T1 measurements from MOLLI are more accurate and reproducible. For participants who have difficulty with holding their breath, the ShMOLLI sequence is recommended.

b) Scan parameters: SA view is recommended for T1 mapping with spatial resolution $\leq 3.0 \times 3.0 \mathrm{~mm}^{2}$, slice thickness $\leq 8.0 \mathrm{~mm}$, and slice gap $\leq 4.0 \mathrm{~mm}$. Imaging should contain at least five slices and 8-12 T1 weighted images (Messroghli et al. 2017). Signals are collected at the end of the diastole with ECG trigger on.

c) Contrast: $\mathrm{T} 1$ represents the time constant of the recovery of longitudinal magnetization (spin-lattice relaxation). A series of signals with different inversion recovery times are collected to fit the pixel-wise $\mathrm{T} 1$ value according to the longitudinal relaxation time attenuation curve (Fig. 4). T1 mapping is often used to quantify the degree of myocardial fibrosis. 


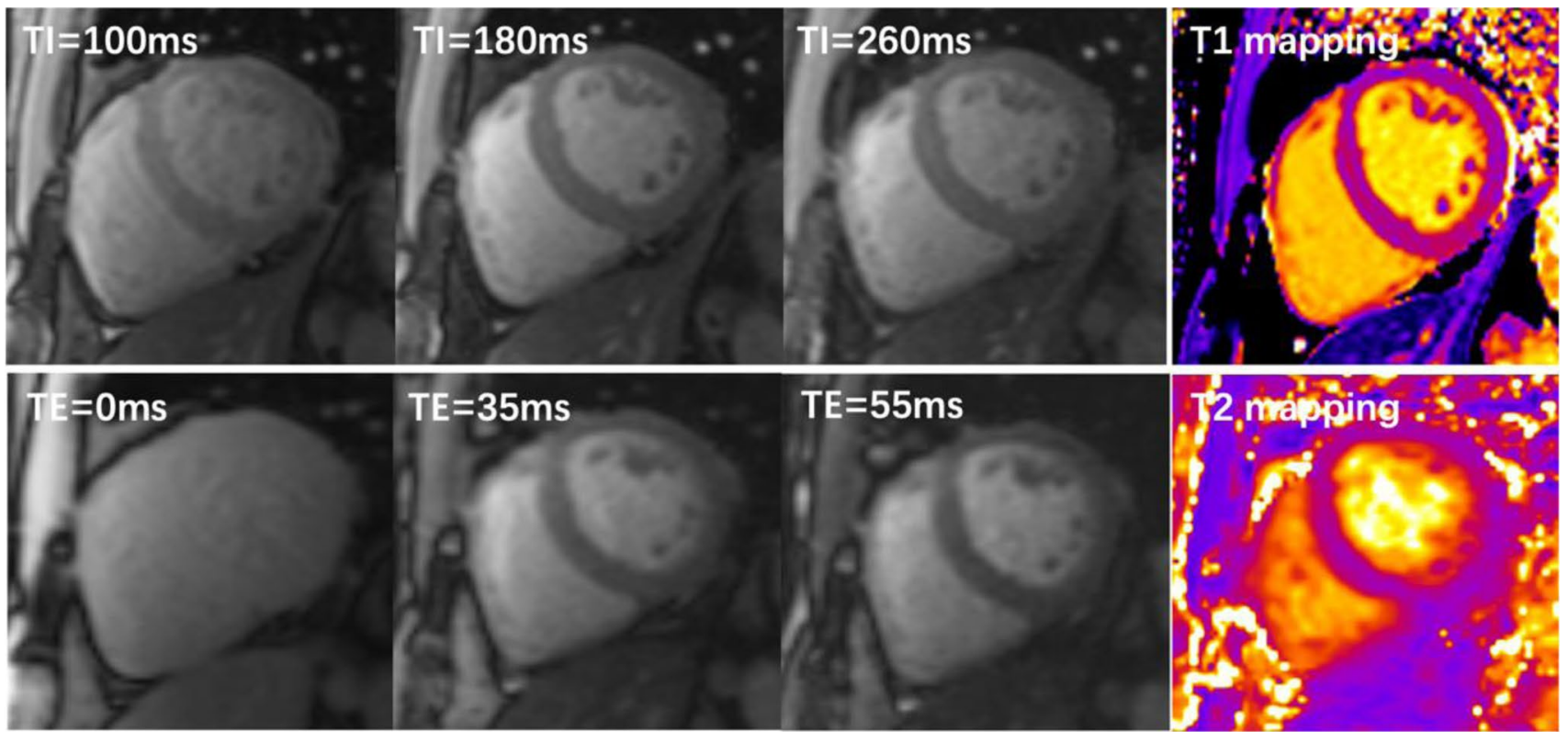

Fig. 4 Example of myocardial T1 (upper row) and T2 mappings (bottom row) acquired with True-FISP

d) Corresponding phenotypes: native T1 mapping can be obtained without the injection of contrast agents. The average and standard deviation of T1 values in 17 myocardial segments can be obtained according to the AHA segmentation guidelines (American Heart Association Writing Group on Myocardial Segmentation and Registration for Cardiac Imaging 2002). Enhanced T1 mapping can be obtained if contrast agents are injected.

\section{T2 Mapping}

a) Sequences: the most commonly used sequences for T2 mapping is T2-prepared (T2prep)-TrueFISP and T2prep-TSE. Over recent years, several improvements have been made, including simultaneous T1/T2 mapping (Guo et al. 2019; Akçakaya et al. 2016; Hamilton et al. 2020) and three-dimensional (3D) T2 mapping (Guo et al. 2019; Qi et al. 2019). Considering that twodimensional (2D) T2 mapping is still the most available sequence on clinical scanners, we recommend to use T2prep-TrueFISP as the first choice, while T2prep-TSE should be considered when $B_{0}$ shimming is not adequate.

b) Scan parameters: SA view is recommended for T2 mapping with spatial resolution $\leq 3.0 \times 3.0 \mathrm{~mm}^{2}$, slice thickness $\leq 8.0 \mathrm{~mm}$, and slice gap $\leq 4.0 \mathrm{~mm}$. Imaging should contain at least five slices and 3-5 T2 weighted images. It is highly recommended to copy the geometrical parameters of T1 mapping to T2 mapping to facilitate subsequent analysis. Signals are collected at the end of the diastole with ECG trigger on. c) Contrast: $\mathrm{T} 2$ represents the time constant of the recovery of transversal magnetization (spin-spin relaxation). A series of signals with different echo times (TE) times are collected to fit the pixel-wise $\mathrm{T} 2$ value according to the transverse relaxation time attenuation curve (Fig. 4). T2 mapping reflects the changes in tissue water and is often used to quantify the degree of myocardial edema/ inflammation.

d) Corresponding phenotypes: the average and standard deviation of $\mathrm{T} 2$ values in 17 myocardial segments can be obtained according to the AHA segmentation guideline (American Heart Association Writing Group on Myocardial Segmentation and Registration for Cardiac Imaging 2002).

\section{T2* Mapping}

a) Sequences: T2* mapping is often obtained with multiple-echo gradient-echo (mGRE).

b) Scan parameters: similar to $\mathrm{T} 1$ and $\mathrm{T} 2$ mappings, SA view is recommended for $\mathrm{T} 2 *$ mapping with spatial resolution $\leq 3.0 \times 3.0 \mathrm{~mm}^{2}$, slice thickness $\leq 8.0 \mathrm{~mm}$, and slice gap $\leq 4.0 \mathrm{~mm}$. Imaging should contain at least five slices and 6-8 echoes for subsequent fitting. The echo time can be set between 2 and $18 \mathrm{~ms}$. It is highly recommended to copy the geometrical parameters of T1/ $\mathrm{T} 2$ mapping to $\mathrm{T} 2 *$ mapping. Signals are collected at the end of the diastole with ECG trigger on.

c) Contrast: $\mathrm{T} 2 *$ represents the time constant of the recovery of transversal magnetization in the presence of local field inhomogeneities. T2* mapping technology is nega- 
tively correlated with the iron content in the myocardium. Although $\mathrm{T} 2 *$ mapping is not as commonly used in clinical routines as $\mathrm{T} 1$ and $\mathrm{T} 2$ mappings, it provides information on the myocardial oxygenations due to the different magnetic susceptibility of oxygenated hemoglobin and deoxyhemoglobin, thus making it sensitive to changes in myocardial oxygen content.

d) Corresponding phenotypes: the average and standard deviation of $\mathrm{T} 2 *$ values in 17 myocardial segments can be obtained according to the AHA segmentation guideline (American Heart Association Writing Group on Myocardial Segmentation and Registration for Cardiac Imaging 2002).

\section{Quantitative Flow (Q-Flow) Imaging}

a) Sequences: Q-flow imaging can be performed using 2D phase-contrast gradient echo (PC-GRE) sequence (Thunberg and Kähäri 2011). It is often combined with cine imaging to obtain the flow velocity of different phases in the cardiac cycle (Fig. 5). Moreover, Q-flow imaging can be implemented using 3D PC-GRE sequence [namely four-dimensional flow (4D-flow)]. However, due to its relatively long scan time (>10 min), we classified the $4 \mathrm{D}$-flow as a Category III sequence, which will be discussed in "4D-Flow Imaging". b) Scan parameters: the imaging plane should be perpendicular to the flow direction with spatial resolution $\leq 2.0 \times 2.0 \mathrm{~mm}^{2}$, slice thickness $\leq 8.0 \mathrm{~mm}$, and slice gap $\leq 4.0 \mathrm{~mm}$. The cardiac cycle is usually segmented into 15-25 phases with a temporal resolution of less than $50 \mathrm{~ms}$. The flow encoding is recommended to be set to $120 \%$ of the maximum flow rate in the target vessel. The most commonly scanned positions include the aortic valve, ascending aortic arch, descending aortic arch and pulmonary artery, etc.

c) Contrast: the amount of phase shift is proportional to the first-order flow velocity. By repeating two flow-sensitive gradient encodings in the opposite directions, PC-GRE can be used to quantify the blood flow velocity by subtraction of the opposite phases. Amplitude map (with or without background suppression) and phase map can be obtained from Q-flow imaging.

d) Corresponding phenotypes: the Q-flow imaging is able to obtain the phenotypes of net flow and velocity, reflux score, systolic and diastolic flow velocity.

\section{Coronary Imaging}

a) Sequences: the most commonly used sequences for coronary imaging are the TrueFISP and FLASH (Fig. 6). The former one can be used without the injection of contrast
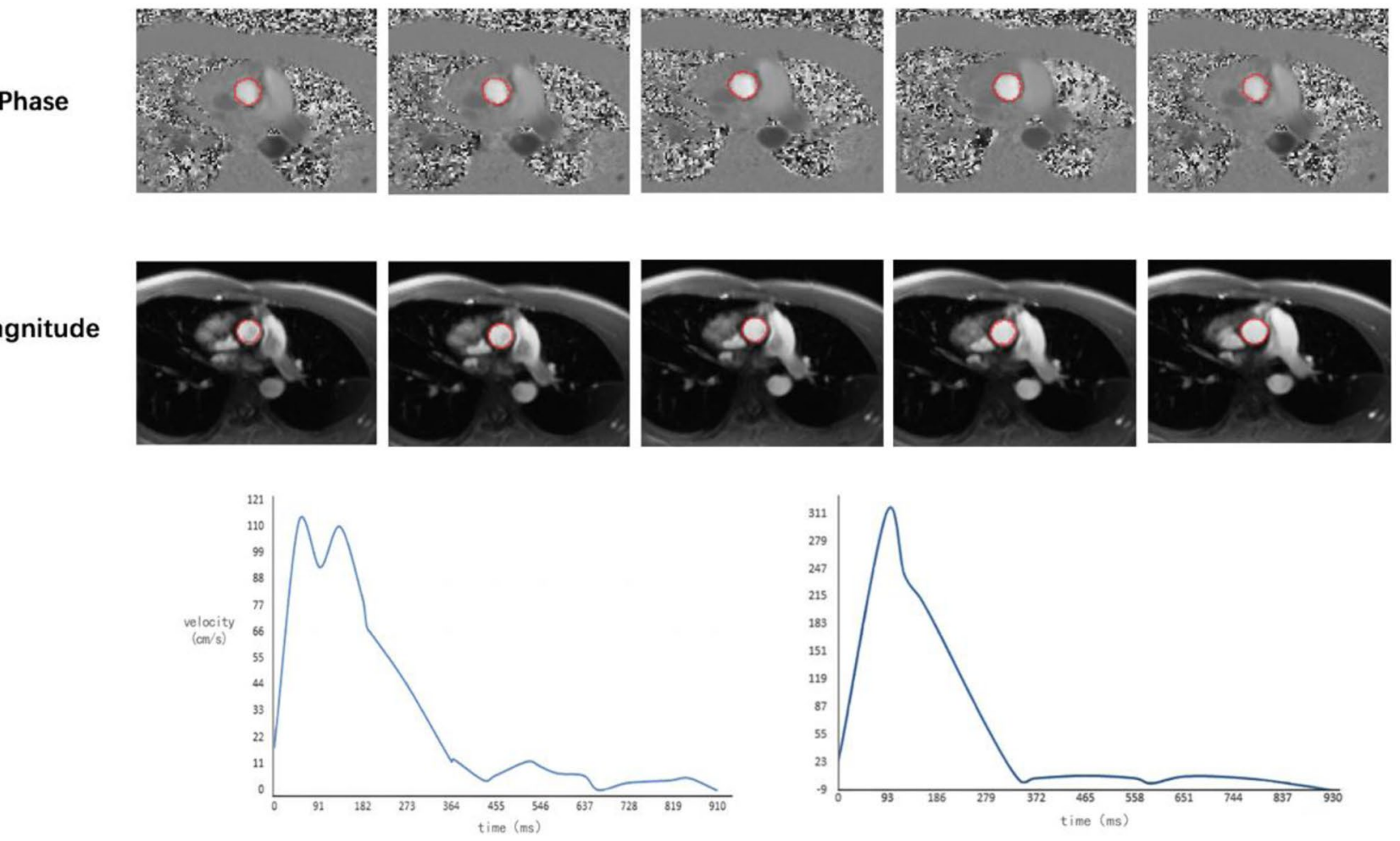

Fig. 5 Example of 2D Q-flow imaging (phase and magnitude images) of the aortic valve and the corresponding flow and velocity measurements 

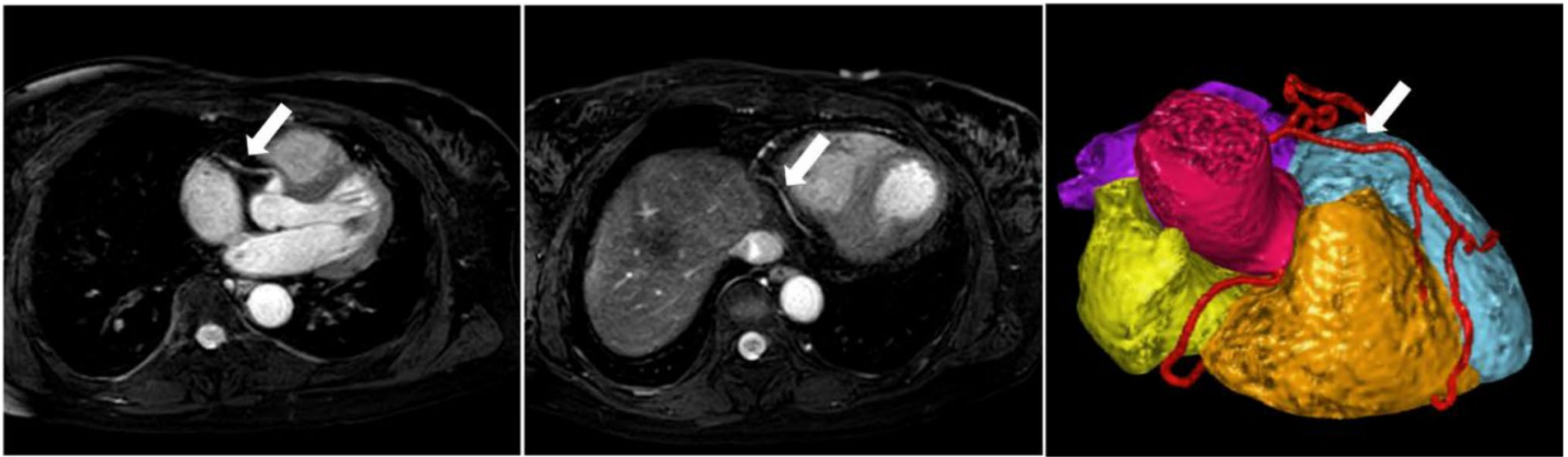

Fig. 6 Example of coronary angiography and the 3D visualization image (white arrows)

agents, which is recommended as the first choice for cohort studies.

b) Scan parameters: coronary imaging can be performed with "target location" or whole heart scan modes. The target location method requires an experienced operator to find the best imaging planar according to the shape of the coronary artery branches. A whole-heart scan covers the entire heart with 3D isotropic imaging. In order to ensure consistency among different scans, it is recommended to perform whole-heart scans with spatial resolution $\leq 1.0 \times 1.0 \times 1.0 \mathrm{~mm}^{3}$. Fat suppression should be performed to enhance the coronary signal.

c) Contrast: post-processing, including maximum intensity projection (MIP), volume reconstruction (VR), and multi-planar reconstruction (MPR), can be used to provide better visualizations of coronary structure, shape, length, and similar. d) Corresponding phenotypes: the coronary imaging is able to measure phenotypes of diameter, length, and curvature of the coronary artery. In order to obtain more detailed phenotypes, it is recommended to perform coronary segment measurements according to the AHA guideline (American Heart Association Writing Group on Myocardial Segmentation and Registration for Cardiac Imaging 2002).

\section{Category III}

\section{D-Flow Imaging}

a) Sequences: 4D-flow imaging is performed using PC FLASH or TrueFISP with 3D perpendicular flow-encodings (Fig. 7).
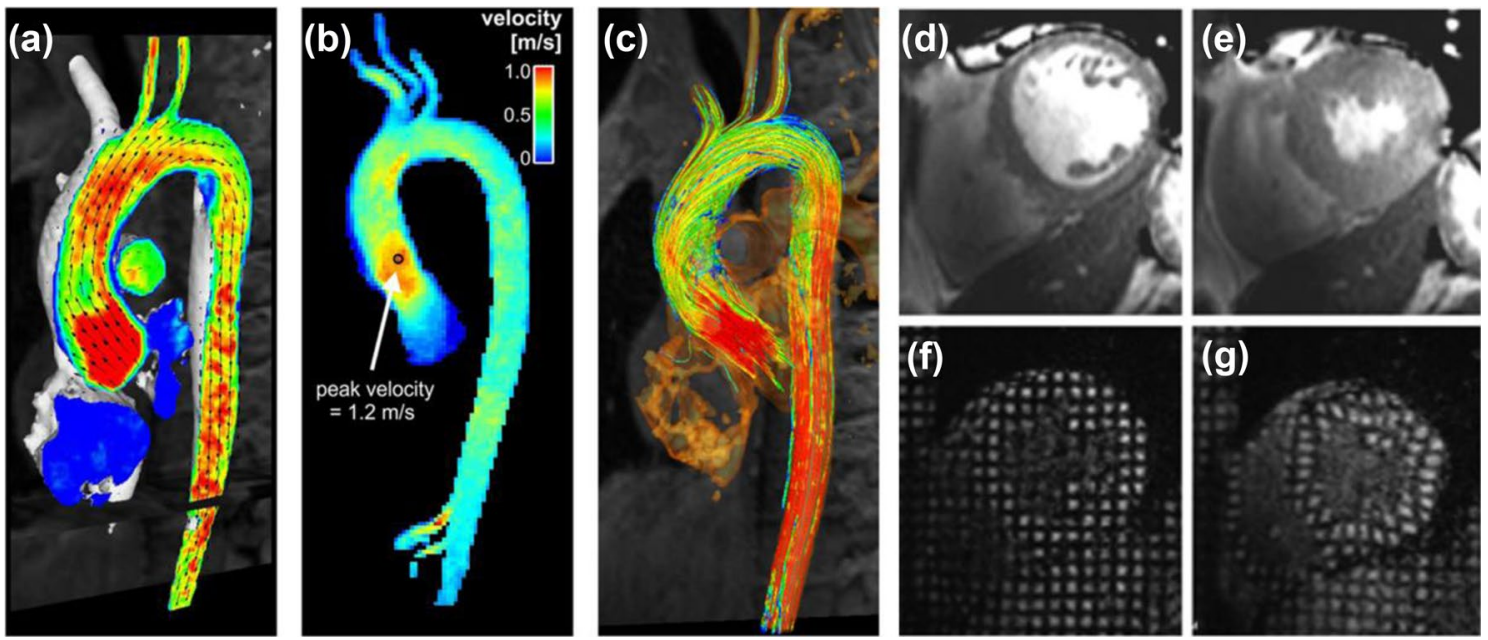

Fig. 7 Examples of 4D-flow imaging with a a color-coded flow speed and a graph of velocity vectors which displays the speed and direction of blood velocity in black arrows; $\mathbf{b}$ a maximum intensity projection image of flow speed; $\mathbf{c}$ streamlines instantaneously tangent to the velocity vector field; $\mathbf{d}$, e cine imaging during the end-diastole and end-systole phases; and $\mathbf{f}, \mathbf{g}$ tagged imaging during the end-diastole and end-systole phases. Adapted from Dyverfeldt et al. (2015) and Hollingsworth et al. (2012) 
b) Scan parameters: isotropic 3D imaging is recommended with spatial resolution $\leq 3.0 \times 3.0 \times 3.0 \mathrm{~mm}^{3}$ and temporal resolution $\leq 40 \mathrm{~ms}$. Retrospective ECG gating and respiratory navigation are used. The speed coding ranges between 150 and $180 \mathrm{~cm} / \mathrm{s}$ according to different anatomies (Dyverfeldt et al. 2015; Markl et al. 2012).

c) Contrast: 4D flow technology is an extension of 2D Q-flow imaging. By applying phase-sensitive gradients on multi-directional blood flows, 3D hemodynamic parameters can be obtained, which can then be used for cardiac (Carlsson et al. 2012), aorta (Bock et al. 2011), and pulmonary artery (Barker et al. 2015).

d) Corresponding phenotypes: 4D-flow imaging can provide measurements of blood flow, velocity, flow, vascular wall shear stress (WSS), turbulent kinetic energy (TKE), pressure gradient (PG), and pulse wave velocity (PWV) within the scan field-of-view (Markl et al. 2016).

\section{Tagged Imaging}

a) Sequences: CMR tagged imaging applies a spatial modulation of magnetization pulse before the cardiac movie imaging starts to perform grid-like marking on the myocardium (Zerhouni et al. 1988). Similar to cine imaging, the most commonly used readouts are segmented TrueFISP and the FLASH.

b) Scan parameters: it is recommended to perform CMR tagged imaging with spatial resolution $\leq 2.0 \times 2.0 \mathrm{~mm}^{2}$, slice thickness $\leq 8.0 \mathrm{~mm}$, slice gap $\leq 4.0 \mathrm{~mm}$, time resolution $\leq 50 \mathrm{~ms}$, and $15-25$ cardiac cycles.

c) Contrast: the CMR tagged imaging applies a set of vertical pre-saturation pulses before cine imaging, which generates multiple interleaved lines or squares on the myocardium. The tagged myocardial segments can be used to evaluate the local mechanical function of the heart. The lines/squares are regularly arranged at the end of the diastole but deformed during the systolic phase. The contraction of the myocardium can be evaluated by observing the morphological changes of the lines/ squares.

d) Corresponding phenotypes: CMR tagged imaging can provide measurements of myocardial strain and strain rate.

\section{Perfusion Imaging}

a) Sequences: the most commonly used sequences are Turbo-FLASH, TrueFISP, and GRE-echo planar imaging (EPI) with T1-weighting (Fig. 8).

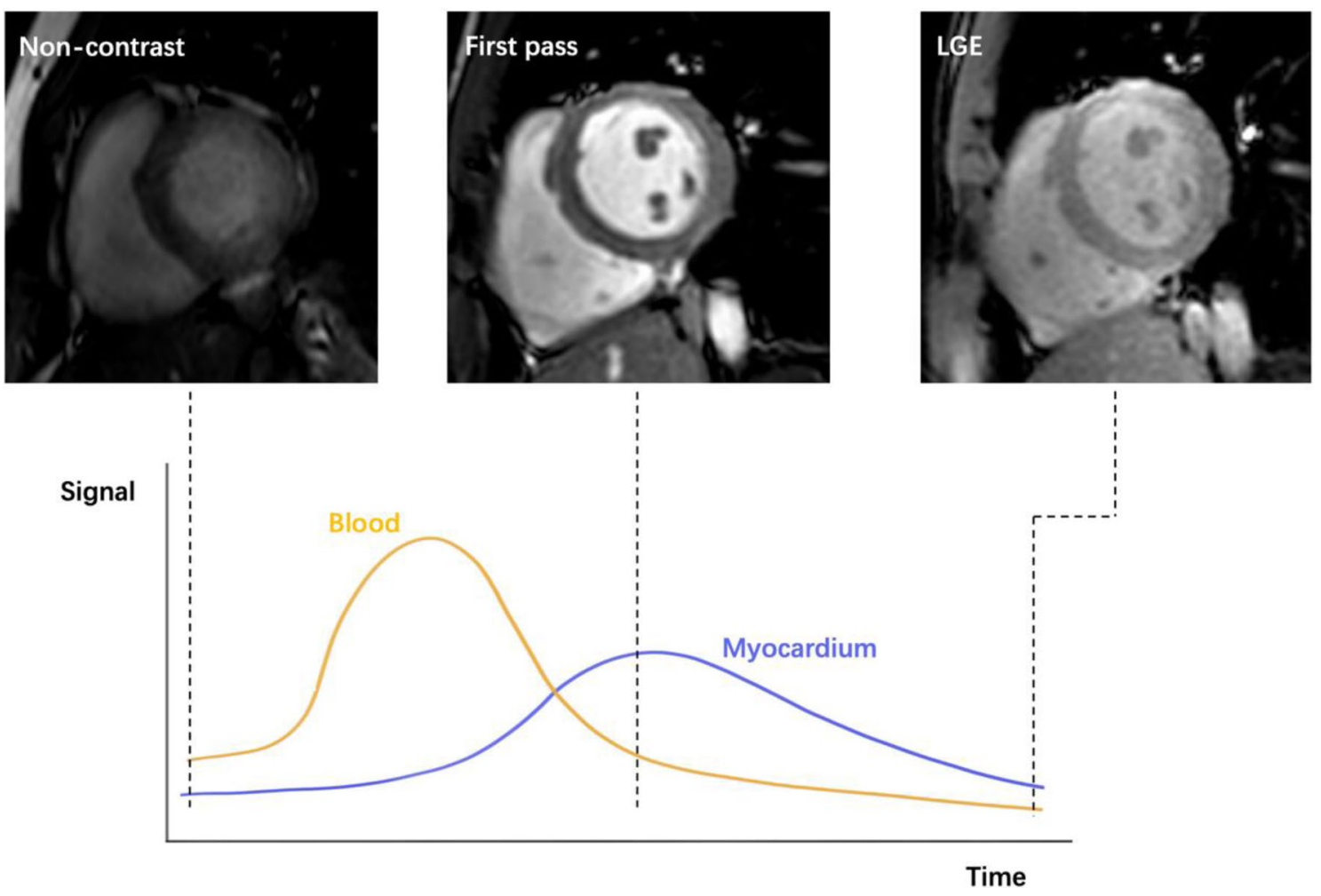

Fig. 8 Examples of perfusion and LGE imaging. Images were acquired before contrast enhancement, during the peak of the enhanced signal, and 10-15 min after contrast agent injection 
b) Scan parameters: SA view is recommended for perfusion imaging with spatial resolution $\leq 3.0 \times 3.0 \mathrm{~mm}^{2}$, slice thickness $\leq 10.0 \mathrm{~mm}$, slice gap $\leq 4.0 \mathrm{~mm}$, and temporal resolution $\leq 125 \mathrm{~ms}$. Gadolinium contrast agents are injected with a dose of $0.1 \sim 0.2 \mathrm{mmol} / \mathrm{kg}$ body weight.

c) Contrast: after the injection of contrast agents, the right ventricle shows first, followed by the left ventricle and myocardium. Myocardial perfusion imaging with stress is often required to increase the sensitivity of myocardial ischemia detection. Both exercise and drug loading can be applied for stress perfusion imaging. The exercise loading needs to be equipped with compatible devices in the MR scan room. Drug loading is based on vasodilators such as adenosine or dipyridamole. This approach has the advantages of simple operation and good repeatability, thus is recommended for stress imaging (Ichikawa et al. 2003).

d) Corresponding phenotypes: by analyzing the signal intensity curve of perfusion imaging, the semi-quantitative parameters such as peak signal intensity, time to peak, and average arrival time can be measured.

\section{Late Gadolinium Enhancement (LGE) Imaging}

a) Sequences: the commonly used sequences are TrueFISP, the rapid inversion recovery (IR-TFE), and the phase-sensitive inversion recovery (PSIR) gradient-echo sequence (GRE) (Hundley et al. 2010; Friedrich et al. 2009).

b) Scan parameters: it is recommended to perform SA scan at 10-15 min after the injection of the contrast agent. To suppress the myocardial signal, inversion recovery (TI) time is set to the T1 relaxation time of the myocardium. LGE imaging can be performed with spatial resolution $\leq 3.0 \times 3.0 \mathrm{~mm}^{2}$, slice thickness $\leq 8.0 \mathrm{~mm}$, slice gap $\leq 4.0 \mathrm{~mm}$.

c) Contrast: during the delayed phase, the normal myocardium shows a low signal since the gadolinium has been cleared. A high signal can be seen in the necrotic myocardium since the residual gadolinium shortens the $\mathrm{T} 1$ relaxation of the tissue. A delayed enhanced cardiac scan can be used to evaluate the myocardial viability and to identify necrotic fibers and scars.

d) Corresponding phenotypes: according to the AHA segmentation standard, the enhanced $\mathrm{T} 1$ values from 17 myocardial segments can be obtained. The extracellular volume fraction (ECV) can be calculated from native $\mathrm{T} 1$, enhanced T1, and the estimated blood cell volume. ECV reflects the tissue fraction that is not occupied by cardiomyocytes.

\section{Diffusion Tensor Imaging (DTI)}

a) Sequences: the recommended sequence for cardiac DTI is stimulated echo pulse (STE) with the signal acquisition within two consecutive cardiac cycles (Mekkaoui et al. 2017) (Fig. 9).

b) Scan parameters: the cardiac DTI is recommended to be performed with spatial resolution $\leq 3.0 \times 3.0 \mathrm{~mm}^{2}$, slice thickness $\leq 8.0 \mathrm{~mm}$, slice gap $\leq 4.0 \mathrm{~mm}$, dispersion gradient directions $\geq 6$, repetition times $\geq 6, b$ values between 300 and $600 \mathrm{~s} / \mathrm{mm}^{2}$ (Nielles-Vallespin et al. 2013). Both SA and $4 \mathrm{CH}$ views can be scanned.

c) Contrast: the fiber tracking based on cardiac DTI can provide unique information about myocardial microstructures, reflecting the tissue integrity. It is currently the only way to evaluate cardiac microstructures in vivo. Normally, endocardial fibers are distributed in a positive spiral, while epicardial muscle fibers are distributed in a reverse spiral. This microstructure is related to the mechanical and electrophysiological functions of the heart.

d) Corresponding phenotypes: the measurable phenotypes include mean diffusivity (MD), fractional anisotropy (FA), and helix angle (HA). Segmented measurements can be conducted according to the AHA guidelines (American Heart Association Writing Group on Myocardial Segmentation and Registration for Cardiac Imaging 2002).

\section{MR Spectroscopy (MRS)}

a) Sequences: currently, the most widely used sequences for MRS are the stimulated echo acquisition mode (STEAM) and point-resolved spectroscopy (PRESS). There are many other spectroscopy imaging techniques; however, as they are still under development, they were not included in this paper (Bizino et al. 2014; Ewijk et al. 2015) (Fig. 10).

b) Scan parameters: single-voxel MRS is recommended with voxel size $\leq 6.0 \times 6.0 \times 6.0 \mathrm{~cm}^{3}$. The region of interest (ROI) can be put on the left ventricle. The scan needs to be repeated 30 to 80 times to get enough SNR. It is recommended to suppress water and fat signals in ${ }^{1} \mathrm{H}$ MRS. Motion compensation is also recommended.

c) Contrast: the most widely used MRS include ${ }^{31} \mathrm{P}$-MRS and ${ }^{1} \mathrm{H}-\mathrm{MRS}$. Since ${ }^{31} \mathrm{P}-\mathrm{MRS}$ requires special coils and is not widely available in clinical scanners, we recommend the use of ${ }^{1} \mathrm{H}$-MRS for cohort studies. It can also be considered by research centers equipped to perform ${ }^{31} \mathrm{P}-\mathrm{MRS}$.

d) Corresponding phenotypes: adenosine triphosphate (ATP) and creatine phosphate (PCr) contents, as well as 

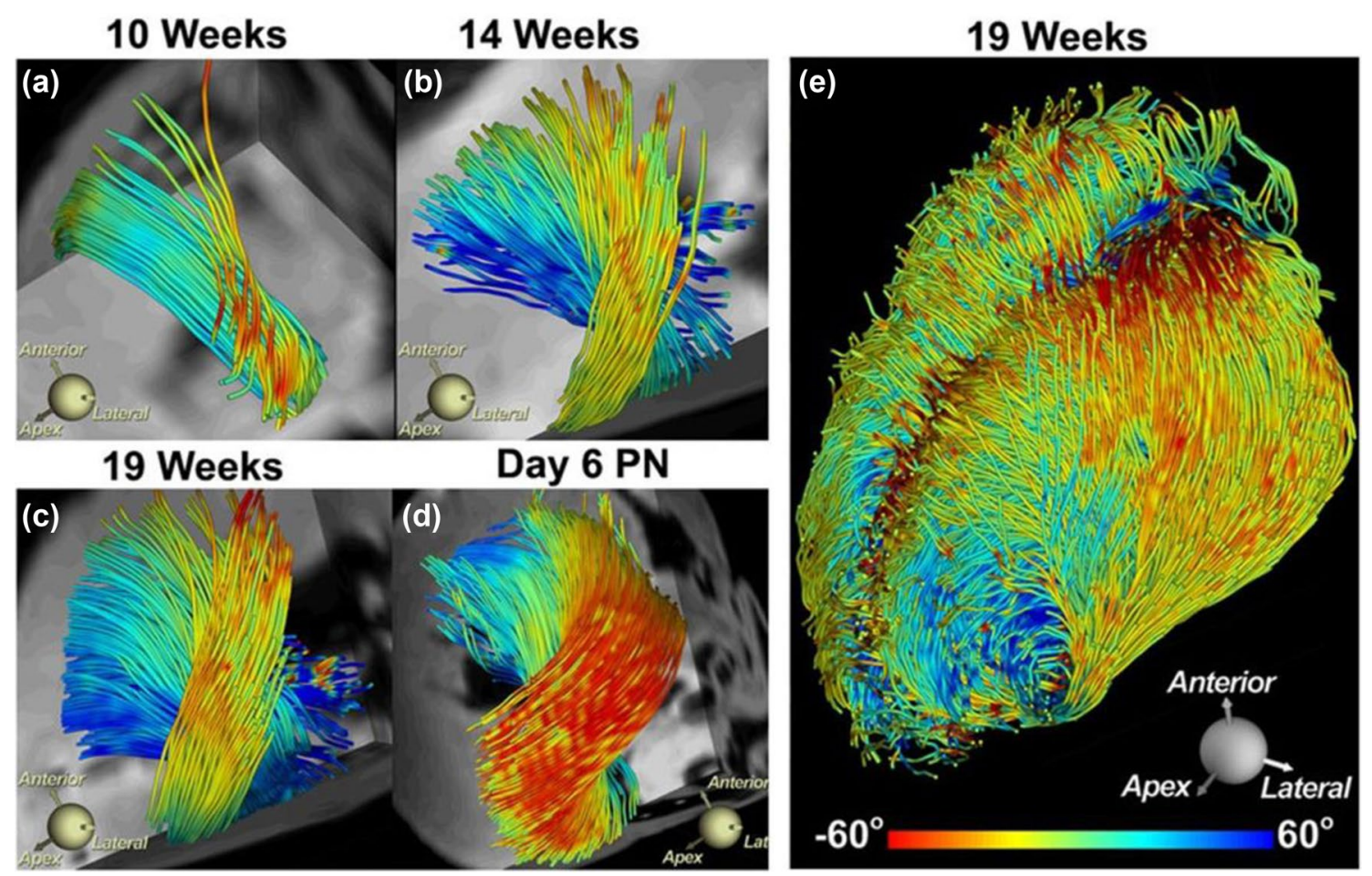

Fig. 9 Examples of DTI and the myofiber architecture in the human fetal heart during different time points. Adapted from Mekkaoui et al. (2013)
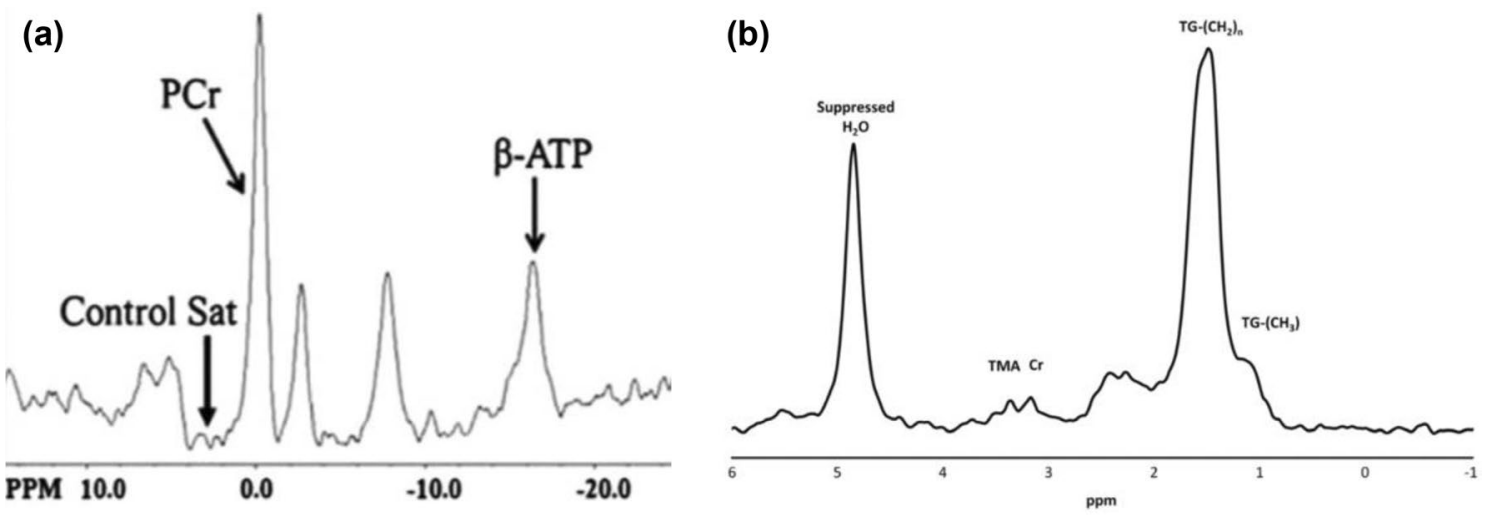

Fig. 10 Examples of in vivo $\mathbf{a}^{1} \mathrm{H}-\mathrm{MRS}$ and $\mathbf{b}{ }^{31} \mathrm{P}-\mathrm{MRS}$. Adapted from van der Meer et al. (2005)

PCr/ATP ratio, can be obtained from ${ }^{31} \mathrm{P}-\mathrm{MRS}$. Creatine and triglyceride content can be obtained from ${ }^{1} \mathrm{H}$-MRS.

\section{Image Reconstruction Method}

Since Cartesian sampling of $k$-space is still the most widely used approach, the image reconstruction can be conducted with conventional parallel imaging techniques (i.e., SENSE and GRAPPA). Compressed sensing (CS) can also be considered to accelerate the imaging speed. For applications of dynamic imaging (i.e., cine, PC-cine, 4D-flow, tagging, and perfusion imaging), $k$ - $t$ BLAST (Sinha et al. 2010), $k$ - $t$ SENSE (Samsonov et al. 2005), and $k$ - $t$ PCA (Pedersen et al. 2009) are available options to further improve the temporal and spatial resolutions. 


\section{Image Quality Control}

\section{Respiratory Motion Control}

Respiratory movement can be compensated by the breathhold or respiratory-gating (Biglands et al. 2012). The breathhold time is often set to $12-25 \mathrm{~s}$ and may be shorter for patients with cardiopulmonary diseases. Respiratory gating can be realized by tracking the movement through a belt or through the intrinsic diaphragm navigation signals. Signals acquired at the end of diastole are used for most sequences unless otherwise specified.

\section{Cardiac Motion Control}

Cardiac movement is very complex, including a contraction in the LA direction, radial contraction, and rotation in the SA direction. Although several sequences can be conducted in a single heartbeat, most sequences still need to fill the full $k$-space with signals from multiple cardiac cycles. The commonly used ECG gating can be conducted in a prospective or retrospective way. Pulse wave gating can replace ECG gating if the delay can be accurately estimated (Fushimi et al. 2012).

Some retrospective motion correction algorithms can be introduced to improve the image quality during the image reconstructions. Among them, several deep learning based algorithms have achieved good performances for motion correction (Duffy et al. 2021; Gong et al. 2020; Wang et al. 2020). Deep learning based quality control algorithms are also useful for large cohort studies (Sujit et al. 2019; Samani et al. 2020; Küstner et al. 2018). It is necessary to ensure the consistency of image reconstruction algorithms in the cohort studies so as to reduce the variation among groups. It is recommended to record the raw data for future multi-center studies (Woodard et al. 2006).

\section{CMR Derived Phenotypes}

Since the cardiac functionality is complex, it requires standardized CMR imaging, as well as standardized phenotype extraction. Herein, we divided the commonly used cardiac phenotypes into five types and provided descriptions and suggestions for measurements (Tables 3 and Table 4).

1. Morphological Phenotypes

Morphological phenotypes include the cavity volume, mass, wall thickness, cavity diameter, vessel diameter, sectional vessel area, and vessel curvatures. Among them, the measurements of volume, mass, and cavity diameter should be independently conducted within four chambers, and both end-diastolic and end-systolic measurements should be reported. The wall thickness is recommended to be measured within 17 segments according to the AHA guidelines (American Heart Association Writing Group on Myocardial Segmentation and Registration for Cardiac Imaging 2002). It is also recommended to measure phenotypes of vessel diameter, sectional area, and curvature in the coronary artery and aorta according to the segmentation criteria described below.

2. Functional Phenotypes

Functional phenotypes include stroke volume, ejection fraction, cardiac output, mitral valve regurgitation, cardiac index, end-diastolic volume index, end-systolic volume index, stroke volume index, ventricular mass index, and heart rate. A four-chamber analysis should be performed for all the functional phenotypes other than mitral valve regurgitation and heart rate. The body surface area used in all index calculations is estimated based on the height and weight of the volunteers, while the heart rate is provided by the ECG recorded during scans.

3. Tissue Characteristics Phenotypes

Tissue characteristics phenotypes can be obtained by the measurements of $\mathrm{T} 1, \mathrm{~T} 2, \mathrm{~T} 2 *$, myocardial strain and strain rate. Myocardial strain and strain rate are measured with CMR tagged imaging. Usually, only the myocardium of the left ventricle is measured. Segsmentation based measurements should be performed according to the AHA guidelines (American Heart Association Writing Group on Myocardial Segmentation and Registration for Cardiac Imaging 2002).

4. Hemodynamic Phenotypes

Hemodynamic phenotypes include average blood flow, peak flow, average velocity, peak velocity, reflux fraction, WSS, TKE, PG and PWV. Among them, the average blood flow, peak flow, average velocity, peak velocity, and reflux fraction are measured by $2 \mathrm{D}-\mathrm{PC}$ and $4 \mathrm{D}$-flow imaging. If there is enough time, imaging of the aortic valve, aortic arch, and pulmonary artery should also be performed. WSS, TKE, PG, PWV can only be measured by 4D-flow imaging. According to the AHA guideline (American Heart Association Writing Group on Myocardial Segmentation and Registration for Cardiac Imaging 2002), segmentation based measurements are also recommended.

5. Metabolic Phenotypes

Metabolic phenotypes include ECV, peak signal intensity, time to peak, average arrival time, MD, FA, HA, ATP content, $\mathrm{PCr}$ content, $\mathrm{PCr} / \mathrm{ATP}$ ratio, creatine, and triglyceride 


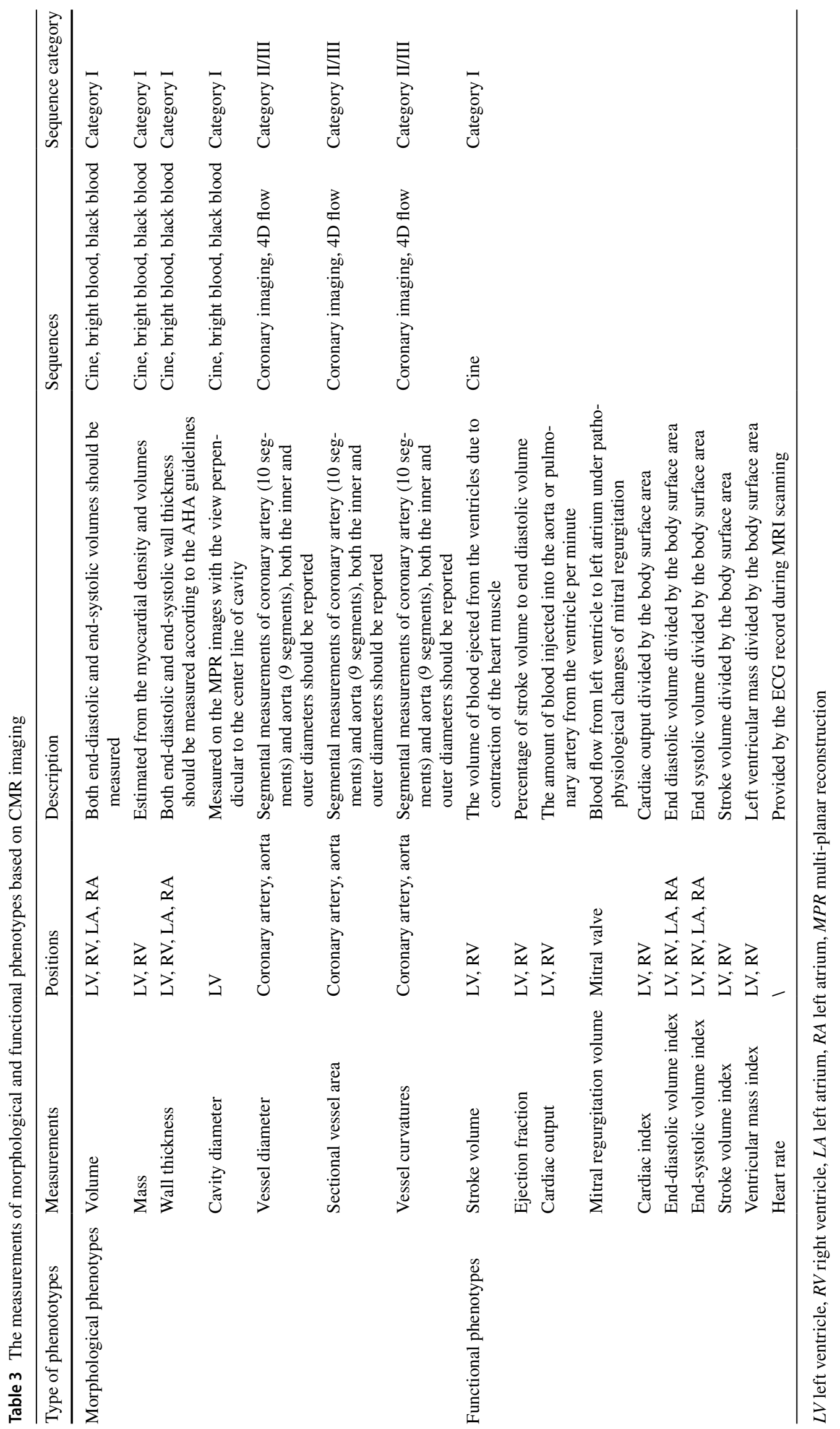


Table 4 Phenotype measurements based on CMR imaging (tissue characteristics, hemodynamic and metabolic phenotypes)

\begin{tabular}{|c|c|c|c|c|c|}
\hline Type of phenototypes & Measurements & Positions & Description & Sequences & Sequence category \\
\hline \multirow[t]{5}{*}{ Tissue characteristics } & $\mathrm{T} 1$ & LV & $\begin{array}{l}\text { Average and standard } \\
\text { deviation of T1 values } \\
\text { in } 17 \text { segments should } \\
\text { be measured accord- } \\
\text { ing to AHA guideline }\end{array}$ & $\begin{array}{l}\text { MOLLI, ShMOLLI, } \\
\text { SASHA }\end{array}$ & Category II \\
\hline & $\mathrm{T} 2$ & LV & $\begin{array}{l}\text { Average and standard } \\
\text { deviation of T2 values } \\
\text { in } 17 \text { segments should } \\
\text { be measured accord- } \\
\text { ing to AHA guideline }\end{array}$ & $\begin{array}{l}\text { T2prep_TrueFISP } \\
\text { T2prep_TSE }\end{array}$ & Category II \\
\hline & $\mathrm{T} 2 *$ & LV & $\begin{array}{l}\text { Average and standard } \\
\text { deviation of T2* } \\
\text { values in } 17 \text { segments } \\
\text { should be measured } \\
\text { according to AHA } \\
\text { guideline }\end{array}$ & mGRE & Category II \\
\hline & Strain & LV & $\begin{array}{l}\text { Myocardial strain dur- } \\
\text { ing systole }\end{array}$ & Tagged cine & Category III \\
\hline & Strain rate & LV & $\begin{array}{l}\text { The change of strain } \\
\text { during systole }\end{array}$ & Tagged cine & Category III \\
\hline \multirow[t]{9}{*}{$\begin{array}{l}\text { Hemodynamic pheno- } \\
\text { types }\end{array}$} & Average velocity & $\begin{array}{l}\text { Aortic valve, aortic } \\
\text { arch, pulmonary } \\
\text { artery }\end{array}$ & $\begin{array}{l}\text { The average velocity of } \\
\text { blood flow along the } \\
\text { vessel }\end{array}$ & 2D PC, 4D flow & Category II/III \\
\hline & Peak velocity & $\begin{array}{l}\text { Aortic valve, aortic } \\
\text { arch, pulmonary } \\
\text { artery }\end{array}$ & $\begin{array}{l}\text { Peak velocity of blood } \\
\text { flow along the vessel }\end{array}$ & 2D PC, 4D flow & Category II/III \\
\hline & Average blood flow & $\begin{array}{l}\text { Aortic valve, aortic } \\
\text { arch, pulmonary } \\
\text { artery }\end{array}$ & $\begin{array}{l}\text { Average blood flow } \\
\text { velocity times diam- } \\
\text { eter }\end{array}$ & 2D PC, 4D flow & Category II/III \\
\hline & Peak flow & $\begin{array}{l}\text { Aortic valve, aortic } \\
\text { arch, pulmonary } \\
\text { artery }\end{array}$ & $\begin{array}{l}\text { Peak blood flow veloc- } \\
\text { ity times diameter }\end{array}$ & 2D PC, 4D flow & Category II/III \\
\hline & Reflux fraction & Mitral valve & $\begin{array}{l}\text { Percentage of mitral } \\
\text { regurgitation to the } \\
\text { total blood }\end{array}$ & 2D PC, 4D flow & Category II/III \\
\hline & WSS & $\begin{array}{l}\text { Aortic arch, pulmonary } \\
\text { artery }\end{array}$ & $\begin{array}{l}\text { Viscous shear forces of } \\
\text { flowing blood acting } \\
\text { tangentially to the } \\
\text { vessel wall }\end{array}$ & 4D flow & Category III \\
\hline & TKE & $\begin{array}{l}\text { Aortic arch, pulmonary } \\
\text { artery }\end{array}$ & $\begin{array}{l}\text { Energy content of } \\
\text { turbulent flow and } \\
\text { direction-independent } \\
\text { measure of intensity } \\
\text { of turbulent velocity } \\
\text { fluctuations }\end{array}$ & 4D flow & Category III \\
\hline & PWV & $\begin{array}{l}\text { Aortic arch, pulmonary } \\
\text { artery }\end{array}$ & $\begin{array}{l}\text { Propagation speed of } \\
\text { systolic pressure pulse } \\
\text { in the arterial system }\end{array}$ & 4D flow & Category III \\
\hline & PG & $\begin{array}{l}\text { Aortic arch, pulmonary } \\
\text { artery }\end{array}$ & $\begin{array}{l}\text { Relative blood pressure } \\
\text { field }\end{array}$ & 4D flow & Category III \\
\hline
\end{tabular}


Table 4 (continued)

\begin{tabular}{|c|c|c|c|c|c|}
\hline Type of phenototypes & Measurements & Positions & Description & Sequences & Sequence category \\
\hline \multirow[t]{11}{*}{ Metabolic phenotypes } & ECV & LV & $\begin{array}{l}\text { The space or volume of } \\
\text { tissue not occupied } \\
\text { by cells }\end{array}$ & MOLLI, LGE & \multirow[t]{11}{*}{ Category III } \\
\hline & Time to peak & LV & $\begin{array}{c}\text { Time from the injection } \\
\text { time to peak intensity }\end{array}$ & Perfusion & \\
\hline & Ratio of peak & LV & $\begin{array}{l}\text { The ratio of peak signal } \\
\text { to initial one }\end{array}$ & Perfusion & \\
\hline & HA & Whole heart & $\begin{array}{l}\text { Fiber distribution } \\
\text { defined by helix angle }\end{array}$ & DTI & \\
\hline & MD & LV & $\begin{array}{l}\text { Average diffusion coef- } \\
\text { ficient of myocardium }\end{array}$ & DTI & \\
\hline & FA & LV & $\begin{array}{l}\text { Anisotropy coefficient } \\
\text { of myocardium }\end{array}$ & DTI & \\
\hline & ATP & ROI & $\begin{array}{l}\text { Adenosine triphosphate } \\
\text { concentration in myo- } \\
\text { cardium }\end{array}$ & ${ }^{31} \mathrm{P}-\mathrm{MRS}$ & \\
\hline & $\mathrm{PCr}$ & ROI & $\begin{array}{l}\text { The concentration of } \\
\text { creatine phosphate in } \\
\text { myocardium }\end{array}$ & ${ }^{31} \mathrm{P}-\mathrm{MRS}$ & \\
\hline & $\mathrm{PCr} / \mathrm{ATP}$ & ROI & $\begin{array}{l}\text { Concentration ratio } \\
\text { of ATP to creatine } \\
\text { phosphate }\end{array}$ & ${ }^{31} \mathrm{P}-\mathrm{MRS}$ & \\
\hline & Creatine & ROI & $\begin{array}{l}\text { Creatine concentration } \\
\text { in myocardium }\end{array}$ & ${ }^{1} \mathrm{H}-\mathrm{MRS}$ & \\
\hline & Triglyceride & ROI & $\begin{array}{l}\text { Triglyceride concentra- } \\
\text { tion in myocardium }\end{array}$ & ${ }^{1} \mathrm{H}-\mathrm{MRS}$ & \\
\hline
\end{tabular}

$L V$ left ventricle, $W S S$ ascular wall shear stress, $T K E$ turbulent kinetic energy, $P G$ pressure gradient, $P W V$ pulse wave velocity, $E C V$ extracellular volume fraction, $M D$ mean diffusivity, $F A$ fractional anisotropy, $H A$ helix angle, $R O I$ region-of-interest, $A T P$ adenosine triphosphate, $P C r$ creatine phosphate

contents. ECV needs to be calculated based on the measurements of myocardial T1 value before and after the injection of contrast agents. Peak signal intensity, time to peak, and average arrival time are extracted from perfusion imaging. MD, FA, and HA are the most important parameters that can be obtained from DTI. ATP, PCr, PCr/ATP ratio, creatine, and triglyceride contents can be obtained from either ${ }^{31} \mathrm{P}$ MRS or ${ }^{1} \mathrm{H}$-MRS. According to the AHA guideline, it is recommended to perform segmentation based measurements (American Heart Association Writing Group on Myocardial Segmentation and Registration for Cardiac Imaging 2002). When segmentation based measurements cannot be applied, the ROIs need to be clearly defined in cohort studies.

\section{Conclusions}

This paper listed some commonly used CMR techniques and the readily available cardiac phenotypes based on the current CMR protocols, hoping to bridge the gap between cardiologists/radiologists and basic science researchers, thus making our contribution to the promotion of paradigm reformation of life science. CMR can provide a one-stop evaluation of the cardiovascular anatomy, functionality, and tissue characteristics information and produce many reproducible phenotypes for cohort studies. The human phenomics project is a large-scale project after the human genome project (HGP), facing much greater challenges than HGP. Human phenomics research is characterized by multi-scale, high-dimensionality, and high-heterogeneity. Although this challenge is great, the potential benefits will be substantial. It can be expected that human phenomics research will bring new paradigms to the biomedical society. In order to achieve such ambitious goal, collaborations of multiple scientific teams are necessary. This paper mainly focuses on the CMR imaging part, but it should be noted that image processing and interpretation are equally important. Given the versatility and variation of quantitative CMR phenotypes, the standardization of those image processing and interpretation is required to 
ensure consistent quality and reproducibility of CMR studies (Schulz-Menger et al. 2013).

It is expected that the development in CMR technology will result in an increasing number of cardiac phenotypes. It should be noticed that there are still many technical challenges in cardiac imaging, such as how to deal with the tradeoff between new technologies and standardized scans, how to reduce the inconsistency among operators, and how to automate the imaging and analysis processes. It is also crucial to keep up with the latest CMR guidelines and criteria. We hope that the abovementioned difficulties will be discussed and solved in the near future.

Authors Contributions CYW performed the analysis, drafted the paper, made critical revisions, and approved the final version. YL, JL, JJ, $\mathrm{XH}, \mathrm{XK}, \mathrm{WC}$ supported in gathering study data, analysis design, and made critical revisions. HW made critical revisions and approved the final version.

Funding This study was funded by the National Natural Science Foundation of China (No. 81971583, No. 62001120), National Key R\&D Program of China (No. 2018YFC1312900, No. 2019YFA0709502), Shanghai Sailing Program (No. 20YF1402400), Shanghai Municipal Science and Technology Major Project (No.2017SHZDZX01, No.2018SHZDZX01), ZJLab and Key Laboratory of Computational Neuroscience and Brain-Inspired Intelligence (Fudan University), Ministry of Education, China.

Data availability The data that support the findings of this study are available on request from the corresponding author.

\section{Declarations}

Code Availability Not applicable.

Conflict of interest The authors declare there is no conflicts of interest regarding the publication of this paper.

Ethics Approval Not applicable.

Consent to Participate Not applicable.

\section{References}

Akçakaya M, Weingärtner S, Basha TA et al (2016) Joint myocardial $\mathrm{T} 1$ and T2 mapping using a combination of saturation recovery and T2-preparation. Magn Reson Med 76(3):888-896. https://doi. org/10.1002/mrm.25975

American Heart Association Writing Group on Myocardial Segmentation and Registration for Cardiac Imaging, Cerqueira MD, Weissman NJ et al (2002) Standardized myocardial segmentation and nomenclature for tomographic imaging of the heart: a statement for healthcare professionals from the Cardiac Imaging Committee of the Council on Clinical Cardiology of the American Heart Association. Circulation 105(4):539-542. https://doi.org/10.1161/ hc0402.102

Bai W, Suzuki H, Huang J et al (2020) A population-based phenome-wide association study of cardiac and aortic structure and function. Nat Med 26(10):1654-1662. https://doi.org/10.1038/ s41591-020-1009

Barker AJ, Roldán-Alzate A, Entezari P et al (2015) Four-dimensional flow assessment of pulmonary artery flow and wall shear stress in adult pulmonary arterial hypertension: results from two institutions. Magn Reson Med 73(5):1904-1913. https://doi.org/10. $1002 / \mathrm{mrm} .25326$

Biglands JD, Radjenovic A, Ridgway JP (2012) Cardiovascular magnetic resonance physics for clinicians: part II. J Cardiovasc Magn Reson 14(1):1-40. https://doi.org/10.1186/1532-429x-14-66

Bizino MB, Hammer S, Lamb HJ (2014) Metabolic imaging of the human heart: clinical application of magnetic resonance spectroscopy. Heart 100(11):881-890. https://doi.org/10.1136/heart jnl-2012-302546

Bock J, Frydrychowicz A, Lorenz R et al (2011) In vivo noninvasive 4D pressure difference mapping in the human aorta: phantom comparison and application in healthy volunteers and patients. Magn Reson Med 66(4):1079-1088. https://doi.org/10.1002/mrm. 22907

Carlsson M, Töger J, Kanski M, Bloch KM, Ståhlberg F, Heiberg E, Arheden H (2011) Quantification and visualization of cardiovascular 4D velocity mapping accelerated with parallel imaging or k-t BLAST: head to head comparison and validation at $1.5 \mathrm{~T}$ and 3 T. J Cardiovasc Magn Reson 13(1):55. https://doi.org/10.1186/ 1532-429X-13-55

Carlsson M, Heiberg E, Toger J et al (2012) Quantification of left and right ventricular kinetic energy using four-dimensional intracardiac magnetic resonance imaging flow measurements. Am J Physiol Heart Circ Physiol 302(4):H893-H900

Chow K, Flewitt JA, Green JD et al (2014) Saturation recovery singleshot acquisition (SASHA) for myocardial T1 mapping. Magn Reson Med 71(6):2082-2095. https://doi.org/10.1002/mrm.24878

Duffy BA, Zhao L, Sepehrband F, Min J et al (2021) Retrospective motion artifact correction of structural MRI images using deep learning improves the quality of cortical surface reconstructions. Neuroimage 230:117756. https://doi.org/10.1016/j.neuroimage. 2021.117756

Dyverfeldt P, Bissell M, Barker AJ et al (2015) 4D flow cardiovascular magnetic resonance consensus statement. J Cardiovasc Magn Reson 17(1):1-19. https://doi.org/10.1016/j.neuroimage.2021. 117756

Ferreira VM, Schulz-Menger J, Holmvang G et al (2018) Cardiovascular magnetic resonance in nonischemic myocardial inflammation: expert recommendations. J Am Coll Cardiol 72(24):3158-3176. https://doi.org/10.1016/j.jacc.2018.09.072

Finn JP, Nael K, Deshpande V et al (2006) Cardiac MR imaging: state of the technology. Radiology 241(2):338-354. https://doi.org/10. 1148/radiol.2412041866

Friedrich MG, Sechtem U, Schulz-Menger J et al (2009) Cardiovascular magnetic resonance in myocarditis: a JACC white paper. J Am Coll Cardiol 53(17):1475-1487. https://doi.org/10.1016/j. jacc.2009.02.007

Fushimi Y, Okada T, Yamamoto A et al (2012) Estimation of the timing of carotid artery flow using peripheral pulse wave-gated MRI. J Magn Reson Imaging 36(2):454-458. https://doi.org/10.1002/ jmri.23665

Gong T, Tong Q, Li Z et al (2020) Deep learning-based method for reducing residual motion effects in diffusion parameter estimation. Magn Reson Med 85(4):2278-2293. https://doi.org/10.1002/ mrm. 28544

Guo R, Chen Z, Herzka DA et al (2019) A three-dimensional freebreathing sequence for simultaneous myocardial T1 and T2 mapping. Magn Reson Med 81(2):1031-1043. https://doi.org/10.1002/ mrm.27466

Hamilton JI, Pahwa S, Adedigba J et al (2020) Simultaneous mapping of $\mathrm{T} 1$ and $\mathrm{T} 2$ using cardiac magnetic resonance fingerprinting 
in a cohort of healthy subjects at $1.5 \mathrm{~T}$. J Magn Reson Imaging 52(4):1044-1052. https://doi.org/10.1002/jmri.27155

Henningsson M, Malik S, Botnar R, Castellanos D, Hussain T, Leiner T (2020) Black-Blood Contrast in Cardiovascular MRI. J Magn Reson Imaging 19:e27399. https://doi.org/10.1002/jmri.27399

Hollingsworth KG, Hodgson T, Macgowan GA et al (2012) Impaired cardiac function in chronic fatigue syndrome measured using magnetic resonance cardiac tagging. J Intern Med 271(3):264270. https://doi.org/10.1111/j.1365-2796.2011.02429.x

Hundley WG, Bluemke DA, Finn JP et al (2010) ACCF/ACR/AHA/ NASCI/ SCMR 2010 expert consensus document on cardiovascular magnetic resonance: a report of the American College of Cardiology Foundation Task Force on Expert Consensus Documents. J Am Coll Cardiol 55(23):2614-2662. https://doi.org/10. 1016/j.jacc.2009.11.011

Ichikawa Y, Sakuma H, Kitagawa K et al (2003) Evaluation of left ventricular volumes and ejection fraction using fast steady-state cine MR imaging: comparison with left ventricular angiography. J Cardiovasc Magn Reson 5(2):333-342. https://doi.org/10.1081/ jcmr-120019422

Jaffe AS, Babuin L, Apple FS (2006) Biomarkers in acute cardiac disease: the present and the future. J Am Coll Cardiol 48(1):1-11. https://doi.org/10.1016/j.jacc.2006.02.056

Jo Y, Kim JJ, Park CH et al (2019) Guideline for cardiovascular magnetic resonance imaging from the Korean Society of Cardiovascular Imaging - part 1: standardized protocol. Korean J Radiol 20(9):1313. https://doi.org/10.3348/kjr.2019.0398

Kelle S, Bucciarelli-Ducci C, Judd RM et al (2020) Society for Cardiovascular Magnetic Resonance (SCMR) recommended CMR protocols for scanning patients with active or convalescent phase COVID-19 infection. J Cardiovasc Magn Reson 22(1):1-4. https:// doi.org/10.1186/s12968-020-00656-6

Kramer CM, Barkhausen J, Flamm SD et al (2008) Standardized cardiovascular magnetic resonance imaging (CMR) protocols, society for cardiovascular magnetic resonance: board of trustees task force on standardized protocols. J Cardiovasc Magn Reson 10(1):1-10. https://doi.org/10.1186/1532-429x-10-35

Kramer CM, Barkhausen J, Flamm SD et al (2013) Standardized cardiovascular magnetic resonance (CMR) protocols 2013 update. J Cardiovasc Magn Reson 15(1):1-10. https://doi.org/10.1186/ 1532-429x-15-91

Kramer CM, Barkhausen J, Bucciarelli-Ducci C et al (2020) Standardized cardiovascular magnetic resonance imaging (CMR) protocols: 2020 update. J Cardiovasc Magn Reson 22(1):1-18. https:// doi.org/10.1186/s12968-020-00607-1

Küstner T, Gatidis S, Liebgott A et al (2018) A machine-learning framework for automatic reference-free quality assessment in MRI. Magn Reson Imaging 53:134-147. https://doi.org/10.1016/j. mri.2018.07.003

Markl M, Frydrychowicz A, Kozerke S et al (2012) 4D flow MRI. J Magn Reson Imaging 36(5):1015-1036. https://doi.org/10.1002/ jmri.23632

Markl M, Schnell S, Wu C et al (2016) Advanced flow MRI: emerging techniques and applications. Clin Radiol 71(8):779-795. https:// doi.org/10.1016/j.crad.2016.01.011

Mekkaoui C, Porayette P, Jackowski MP, Kostis WJ, Dai G, Sanders S, Sosnovik DE (2013) Diffusion MRI tractography of the developing human fetal heart. PLoS ONE 8(8):e72795. https://doi.org/10. 1371/journal.pone.0072795

Mekkaoui C, Reese TG, Jackowski MP et al (2017) Diffusion MRI in the heart. NMR Biomed 30(3):e3426. https://doi.org/10.1002/ nbm. 3426

Messroghli DR, Radjenovic A, Kozerke S, Higgins DM, Sivananthan MU, Ridgway JP (2004) Modified look-locker inversion recovery (MOLLI) for high-resolution T1 mapping of the heart. Magn Reson Med 52:141-146. https://doi.org/10.1002/mrm.20110
Messroghli DR, Moon JC, Ferreira VM et al (2017) Clinical recommendations for cardiovascular magnetic resonance mapping of $\mathrm{T} 1, \mathrm{~T} 2, \mathrm{~T} 2 *$ and extracellular volume: a consensus statement by the Society for Cardiovascular Magnetic Resonance (SCMR) endorsed by the European Association for Cardiovascular Imaging (EACVI). J Cardiovasc Magn Reson 19(1):1-24. https://doi. org/10.1186/s12968-017-0389-8

Nakamura M, Sadoshima J (2018) Mechanisms of physiological and pathological cardiac hypertrophy. Nat Rev Cardiol 15(7):387407. https://doi.org/10.1038/s41569-018-0007-y

Nielles-Vallespin S, Mekkaoui C, Gatehouse P et al (2013) In vivo diffusion tensor MRI of the human heart: reproducibility of breath-hold and navigator-based approaches. Magn Reson Med 70(2):454-465. https://doi.org/10.1002/mrm.24488

Pedersen H, Kozerke S, Ringgaard S et al (2009) k-t PCA: temporally constrained k-t BLAST reconstruction using principal component analysis. Magn Reson Med 62(3):706-716. https://doi.org/ $10.1002 / \mathrm{mrm} .22052$

Piechnik SK, Ferreira VM, Dall'Armellina E, Cochlin LE, Greiser A, Neubauer S, Robson MD (2010) Shortened modified look-locker inversion recovery (ShMOLLI) for clinical myocardial T1-mapping at 1.5 and $3 \mathrm{~T}$ within a 9 heartbeat breathhold. J Cardiovasc Magn Reson 12:69. https://doi.org/10.1186/1532-429x-12-69

Qi H, Bustin A, Cruz G et al (2019) Free-running simultaneous myocardial T1/T2 mapping and cine imaging with 3D whole-heart coverage and isotropic spatial resolution. Magn Reson Imaging 63:159-169. https://doi.org/10.1016/j.mri.2019.08.008

Salerno M, Janardhanan R, Jiji RS, Brooks J, Adenaw N, Mehta B, Yang Y, Antkowiak P, Kramer CM, Epstein FH (2013) Comparison of methods for determining the partition coefficient of gadolinium in the myocardium using T(1) mapping. J Magn Reson Imaging 38(1):217-224. https://doi.org/10.1002/jmri. 23875

Samani ZR, Alappatt JA, Parker D et al (2020) QC-automator: deep learning-based automated quality control for diffusion MR images. Front Neurosci 22(13):1456. https://doi.org/10.3389/ fnins.2019.01456

Schulz-Menger J, Bluemke DA, Bremerich J et al (2013) Standardized image interpretation and post processing in cardiovascular magnetic resonance: Society for Cardiovascular Magnetic Resonance (SCMR) board of trustees task force on standardized post processing. J Cardiovasc Magn Reson 15(1):35. https://doi.org/ $10.1186 / 1532-429 x-15-35$

Sinha N, Saranathan M, Ramakrishnan AG (2010) Improved k-t BLAST for fast fMR imaging. J Magn Reson 204(2):273-280. https://doi.org/10.1016/j.jmr.2010.03.007

Sujit SJ, Coronado I, Kamali A et al (2019) Automated image quality evaluation of structural brain MRI using an ensemble of deep learning networks. J Magn Reson Imaging 50(4):1260-1267. https://doi.org/10.1002/jmri.26693

Thunberg P, Kähäri A (2011) Visualization of through-plane blood flow measurements obtained from phase-contrast MRI. J Digit Imaging 24(3):470-477. https://doi.org/10.1007/s10278-010-9286-5

van der Meer RW, Lamb HJ, Smit JW et al (2012) MR imaging evaluation of cardiovascular risk in metabolic syndrome. Radiology 264:21-37. https://doi.org/10.1148/radiol.12110772

van Ewijk PA, Schrauwen-Hinderling VB, Bekkers SCAM et al (2015) MRS: a noninvasive window into cardiac metabolism. NMR Biomed 28(7):747-766. https://doi.org/10.1002/nbm.3320

Wang CY, Liang YC, Wu Y et al (2020) Correction of out-of-FOV motion artifacts using convolutional neural network derived prior image. Magn Reson Imaging 71:93-102. https://doi.org/10.1016/j. mri.2020.05.004

Wassmuth R, Schulz-Menger J (2011) Cardiovascular magnetic resonance imaging of myocardial inflammation. Expert Rev Cardiovasc Ther 9(9):1193-1201. https://doi.org/10.1586/erc.11.118 
Weingärtner S, Akçakaya M, Basha T et al (2014) Combined saturation/inversion recovery sequences for improved evaluation of scar and diffuse fibrosis in patients with arrhythmia or heart rate variability. Magn Reson Med 71(3):1024-1034. https://doi.org/ $10.1002 / \mathrm{mrm} .24761$

Woodard PK, Bluemke DA, Cascade PN et al (2006) ACR practice guideline for the performance and interpretation of cardiac magnetic resonance imaging (MRI). J Am Coll Radiol 3(9):665676. https://doi.org/10.1016/j.jacr.2006.06.007

Zerhouni EA, Parish DM, Rogers WJ et al (1988) Human heart: tagging with MR imaging - a method for noninvasive assessment of myocardial motion. Radiology 169(1):59-63. https://doi.org/10. 1148/radiology.169.1.3420283 